\title{
The short chain fatty acid sodium butyrate attenuates imiquimod-induced psoriasis-like skin inflammation
}

\author{
A. Bruhs, A. Schwarz and T. Schwarz
}

Department of Dermatology, University Clinics Schleswig-Holstein, Campus Kiel, D-24105 Kiel, Germany

\section{Introduction}

There is solid evidence that in the intestine the microbiome modulates inflammatory reactions and that this regulatory effect is achieved via the expansion and activation of regulatory $T$ cells (Treg). Short-chain fatty acids (SCFA), microbiota-derived bacterial fermentation products including butyrate, propionate and acetate, appear to regulate only in the gut, but also in other outside barrier organs including the skin, the oral cavity, the respiratory and the urogenital tract. Since skin commensals also produce SCFA, we postulated that SCFA might also exert a similar regulatory function in the skin as in the gut. Hence, we studied whether SCFA can suppress immunologically mediated inflammatory reactions. The studies were performed in the murine imiquimod-mediated inflammatory model that is established as a murine model of psoriasis-like skin inflammation. This mode allows further elucidation of the pathogenic mechanisms and evaluation of new therapies in psoriasis.

\section{Results}

Bacterial families identified from sequenced 16S rRNA from mice ears and back skin

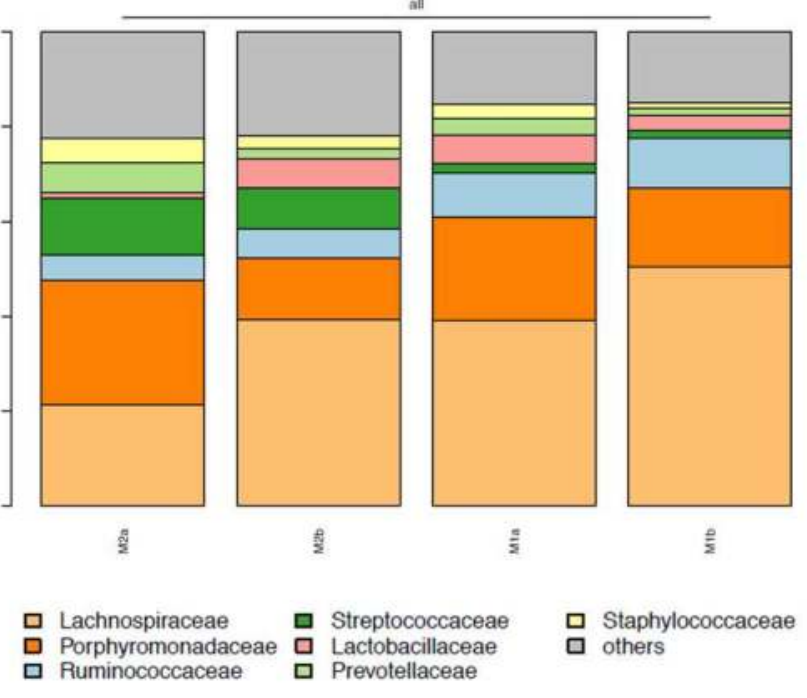

To get an idea whether the cutaneous microbiome includes bacteria which produce SCFA, the microbiome of C57BL/6J mice housed under regular conditions in our anima facility was characterized by 165 rRNA gene sequencing. The microbiome andysis gene analysis revealed the presence of an average of 52 taxa. The main population consisted of members of the Lachnospiraceae, Porphyromonadaceae, Ruminococcaceae, Streptococcacea, Lactobacillaea, Prevotellaceae and Staphylococcaceae (Fig. 1). With the exception of Prevotellaceae, they all belong to the phylum of Firmicutes and the polyphyletic class of Clostridia within the Firmicutes phylum. Strains belonging to Firmicutes were identified as the major butyrate-producing group (Louis and Flint 2009; Pryde et al, 2002). Thus, the majo species identified on mouse skin are potential producers of SCFA.

Fig. 1 colonic Treg homeostasis. There is evidence that commensals control inflammation not

SB contributes to the downregulation of the inflammatory response and thereby might activate cutaneous Treg

Total RNA was isolated from the back skin and ears and qRT-PCR for the transcription of $/ 117$ was performed. $/ 117$ transcripts were significantly upregulated by IMQ which is in accordance with previous observations (van der Fits et al., 2009). SB downregulated $/ 117$ transcription significantly. In turn, transcription of $/ 110$ was remarkably induced by SB. We surmise Treg to be the primary source of IL-10 which was supported by the upregulation of the mRNA of the Treg-specific source of IL-10 which was suppo
transcription factor FoxP3 (Fig. 3a).
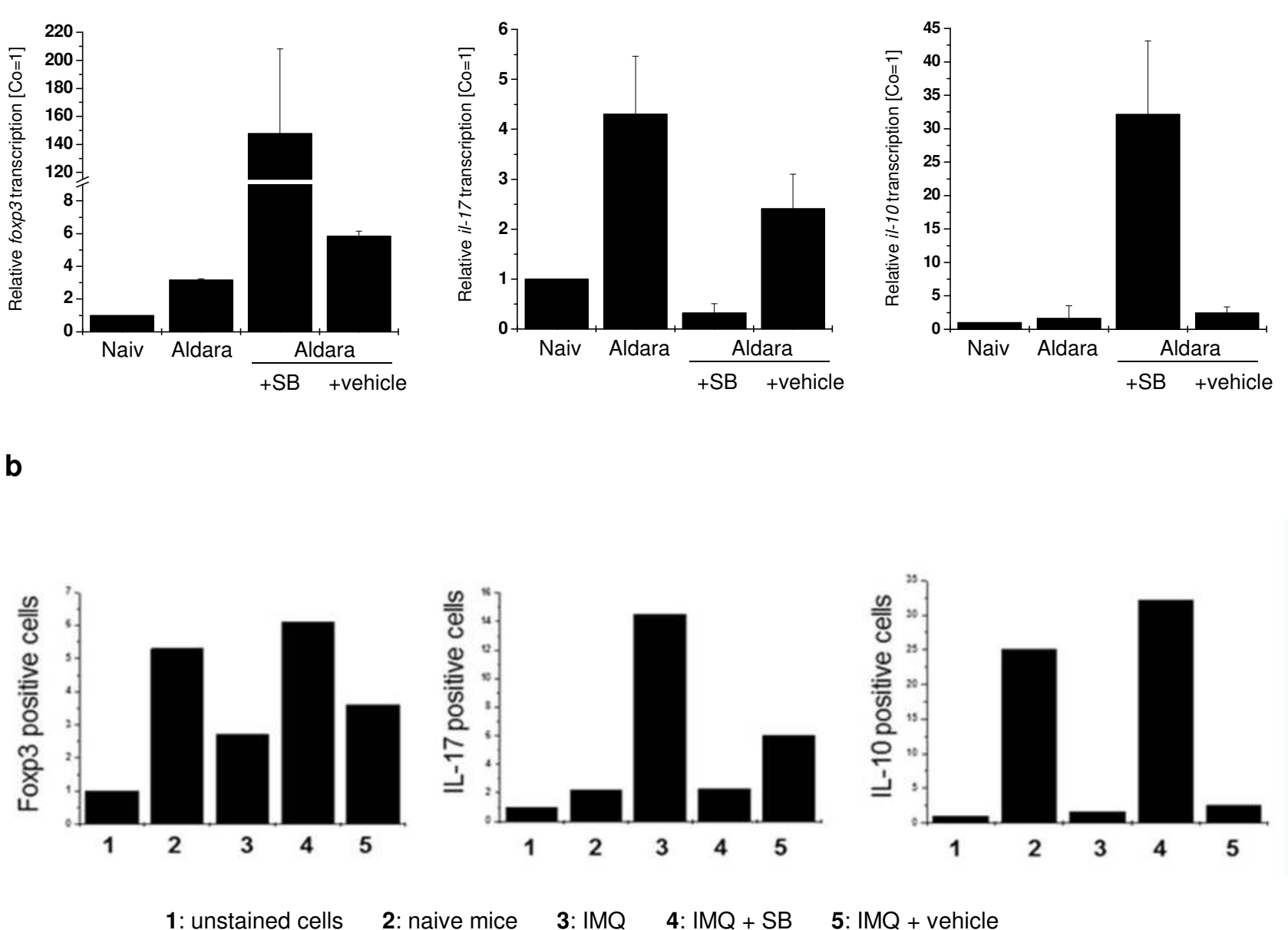

Fig. 3

To get an idea about the cause of the splenomegaly, cells were obtained from spleens and lymph nodes and subjected to FACS analysis. Cells were gated for CD45+ cells and analyzed for the expression of FoxP3, IL-10 and IL-17. The number of IL-17-expressing cells was remarkably enhanced in IMQ-treated mice (Fig. $3 \mathrm{~b}$ ), implying that the splenomegaly may be in part due to the infiltration/expansion of IL-17 expressing lymphocytes. This effect was almost completely prevented by SB. In addition, significantly fewer cells expressing FoxP3 and IL-10 were detected after IMQ administration, suggesting downregulation of Treg. This effect was also reversed by SB

\section{Summary}

The inflammatory response in imiquimod (IMQ)-induced psoriasis-like skin inflammation model is significantly reduced upon administration of SB

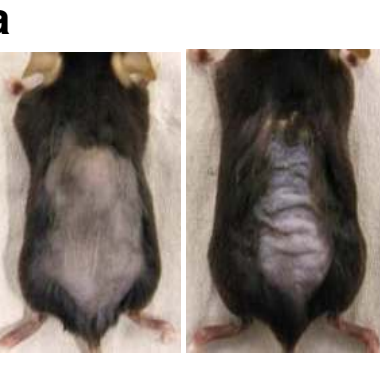

Aldara

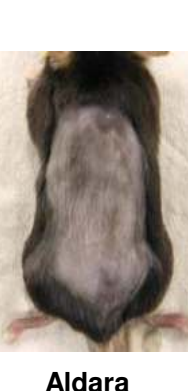

Aldara

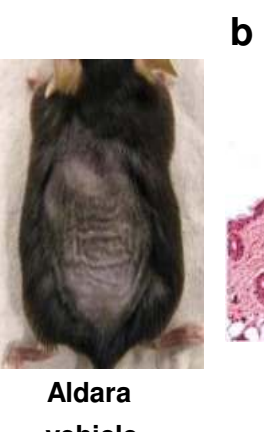

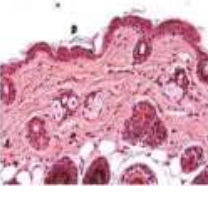

Untreated

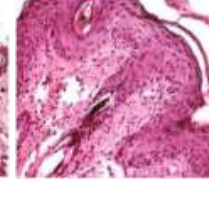

Aldar

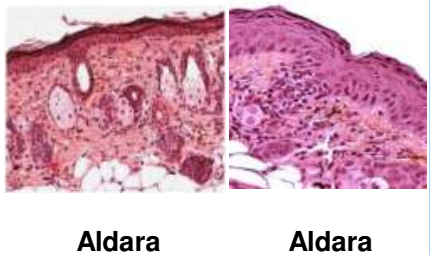

Aldara
Mice were treated with $5 \% \mathrm{IMQ}$ cream (Aldara $\Theta$ ) and received a daily topical dose of $62,5 \mathrm{mg}$ on the shaved backs and ears for 10 days, as described (Alrefai et all., 2016). One group of IMQ-treated mice received topically on the back skin $100 \mu \mathrm{SB}$ (1 mM in a 7:3 mix of 1,2-propandiol:isopropyl alcohol) for 3 days. IMQ-treated mice developed thickening of the skin, erythema and scales. The inflammatory response was significantly reduced upon administration of SB, whereas the vehicle control had no effect (Fig. 2a).

Biopsies were taken, paraffin embedded sections stained with $\mathrm{H} \& \mathrm{E}$ and analyzed histopathologically. IMQ treatment induced acanthosis, hyperkeratosis and an inflammatory infiltrate. These changes were almost completely reversed by SB whereas vehicle had no effect (Fig. 2b).

c

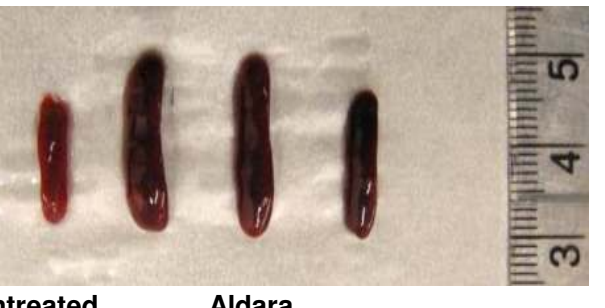

Since IMQ, though only topically applied, appears to induce also systemic immunologic alterations (van der Fits et al., 2009), mice were sacrificed 14 days after initiation of IMQ treatment and spleens were obtained. As described previously, spleens of IMQ treated animals were remarkably enlarged. Splenomegaly was almost completely reversed upon topical application of SB for 3 days (Fig. 2c).
- SCFA sodium butyrate significantly reduces the inflammatory effects in IMQ-induced psoriasis-like skin model.

- Sodium butyrate appears to be a potent immunoregulatory substance which obviously works if applied topically.

- One mode of action by which SB exerts these effects may be the activation/expansion of Treg.

\section{Conclusion}

By virtue of its structure (natural compound, small size, capacity to penetrate into the skin), SB may harbor great therapeutic potential in the treatment of inflammatory skin disorders. 